\title{
The Architecture of Color: Number and Shapes as Measurement and Representation Tools
}

\author{
Michela Rossi • Giorgio Buratti
}

Published online: 21 February 2015

(C) Kim Williams Books, Turin 2015

\begin{abstract}
The paper deals with the geometrical architecture of color models. Many of these models had practical application in industry and the arts. Historically, geometry has been the principal means to explain, measure and represent the phenomenon of color. A 2-year research project on color in industrial design and architecture has led to the development of a new digital model for design applications using generative software (Grasshopper). It aims to show the historical significance of mathematical relationships between color and shape, and the actual applicability of them to digital representation in a dynamic model. The research goal is the parametric modeling of a color solid whose surfaces can be adapted to several geodesic tessellations. A regular polyhedron projected onto the sphere allows the integration of different regular and semi-regular shapes, therefore the comparison of different sets of color with analogous symmetry. The developing process of the model stresses the symmetry of the three primary colors, referring to the RGB screen in the numbers that feature the changing of the hues on the solid's surface, however the number symmetry differs from the first shape.
\end{abstract}

\section{Introduction}

Since very early times, the wonder of color has played an important role in the human approach to the exterior experience of the world. In nature, color is

The paper summarizes only essential references to color models, among the very wide bibliography about color.

M. Rossi $(\bowtie) \cdot$ G. Buratti

Politecnico di Milano, Milan, Italy

e-mail: michela.rossi@polimi.it

G. Buratti

e-mail: giorgio.buratti@polimi.it 
considered a key characteristic. Its main reference is however to the aesthetics of the outside appearance of things, not to the visible expression of their substance, because Western culture relates substance to shape and it conceives measurement, meaning the geometry, as one of the most effective instruments in the knowledge of the physical world. Actually geometry, which is the oldest branch of mathematics, was the first "utility" to visualize and to explain natural phenomena. Because of that it became the instrument by which architects imitated nature's model, that is, the very first reference of design (Rossi 2006).

Nevertheless the marvel of the variety and the beautiful harmonies of color led several to attempt an explication of this phenomenon and actually, after Plato, they used just geometry, meaning shape, to describe and clarify color too. Plane and 3D figures were used as the visible reference of the theoretical concept and as the measurement tool for the screening of pigment mixtures.

A careful survey of many geometrical models presents the continuous variation between colors compared with different theories applied to the arts, industry and design, and shows the great reliability of this intuitive understanding of colour. On one hand, it is interesting to see how different shapes express similar concepts; on the other hand, similar shapes can recall opposite assumptions.

The introduction of spatial references in the Middle Ages by Grosseteste (Silvestrini and Fischer 1997; Marotta 1999) allowed the development of accurate color application, first in painting and dying, then in printing and the creation of color in digital monitors. Later the debut of digital tools in the design process enhanced the role of color in architecture, increasing its importance as an active element in the creation of space, and in the perception and representation of architectural surfaces. Consequently design requires a new attention to the tools that allow and facilitate the choice of and the approach to colors. Color is a complex phenomenon, generated by light on opaque surfaces, and this is translated by pigment into painting. The basic difference between its chemical and physical nature leads to the development of alternative systems in which theoretical models become the tool of practical applications. They always begin with a mix of pigments or lights.

Many theories explain the continuous transition among different hues with reference to simple systems. The color models express nuances in relation to reducing the number of factors that measure quality as quantity expressed by numbers. The theoretical models apply the features of geometry in order to display this complexity accurately. The shape explains, measures and represents the color components (primary colors), the relative amounts in the mixers (secondary colors), the amount of light (brightness) and the intensity (saturation), through the three coordinates of colors. It expresses the color qualities in measurable quantities such as distances and angles. The immediacy of the association between theoretical phenomenon and geometrical model explains, over the centuries, the number of alternatives.

Regular shapes such as the color wheel and the sphere are well rooted in Western culture, thanks to Itten's lessons and others Bauhaus masters who applied them (Rossi 2010). These shapes are representative of a wider sample, more or less regular, expressing the desire to find a common link between the harmony of 
different arts, at first between painting and sculpture/architecture, then between color and shape. The geometry of color is not a curiosity, nor a closed chapter from the past. The old models, some of which have had considerable success over time, still suggest valuable insights for the application to "making".

This paper reports the findings of a long research project that has led to the development of new instruments for color-design, which applies tradition to contemporary needs by digital representation tools. The research demonstrates that color acts as a significant element of drawing, which may apply geometry as a friendly interface to mathematical algorithms.

The structure of the paper follows the development of the research from the history, the comparison and the classification of color models to the design and the improvement of a new digital representation tool.

The content articulates in five paragraphs, before the conclusion:

- Introduction, which explains the relationship between color and geometry.

- Geometry and color, the "architecture" of models that summarizes the history of color models.

- Geometry between theory and practice, which describes the practical application of these models to the arts.

- Color's concepts and patterns, that stresses the relationship between form and color.

- A geometric tool for color design, describing the concept and the development of the digital model for Color Design.

- Conclusion, the actuality of geometry in color-design management.

\section{Geometry and Color, the "Architecture" of Models}

The advent of digital tools in the design process has increased the importance of color, as demonstrated by the increasing attention to color in recent times. It is more and more an active element in the representation of space. Eventually color as the feature element of the shape and design will require an increasing focus to facilitate the representation of colors in the design approach.

This paper explores the outcome of 2 years of research into architectural representation, ${ }^{1}$ using a new color model that has applied topological transformation among shapes. In this way it has developed great adaptability to the different purposes of color representation. Developing the relationships between descriptive geometry and digital representation, it focused its attention on the geometry of color in order to verify the possibility of improving representational tools for the design process. The next step was the implementation of a digital model and experimentation with its applications. The cataloguing and the comparison of the color models

\footnotetext{
1 PRIN 2008, granted by the Ministry of Research and University: "Descriptive Geometry and Digital Representation: Memory and Innovation", general investigator prof. Riccardo Migliari, University of Rome La Sapienza, teams from Italian universities of Genoa, Venice, Udine and Politecnico di Milano (with its local topic 'the relation of shape/color in Design Application of digital tools to color in design and representation').
} 


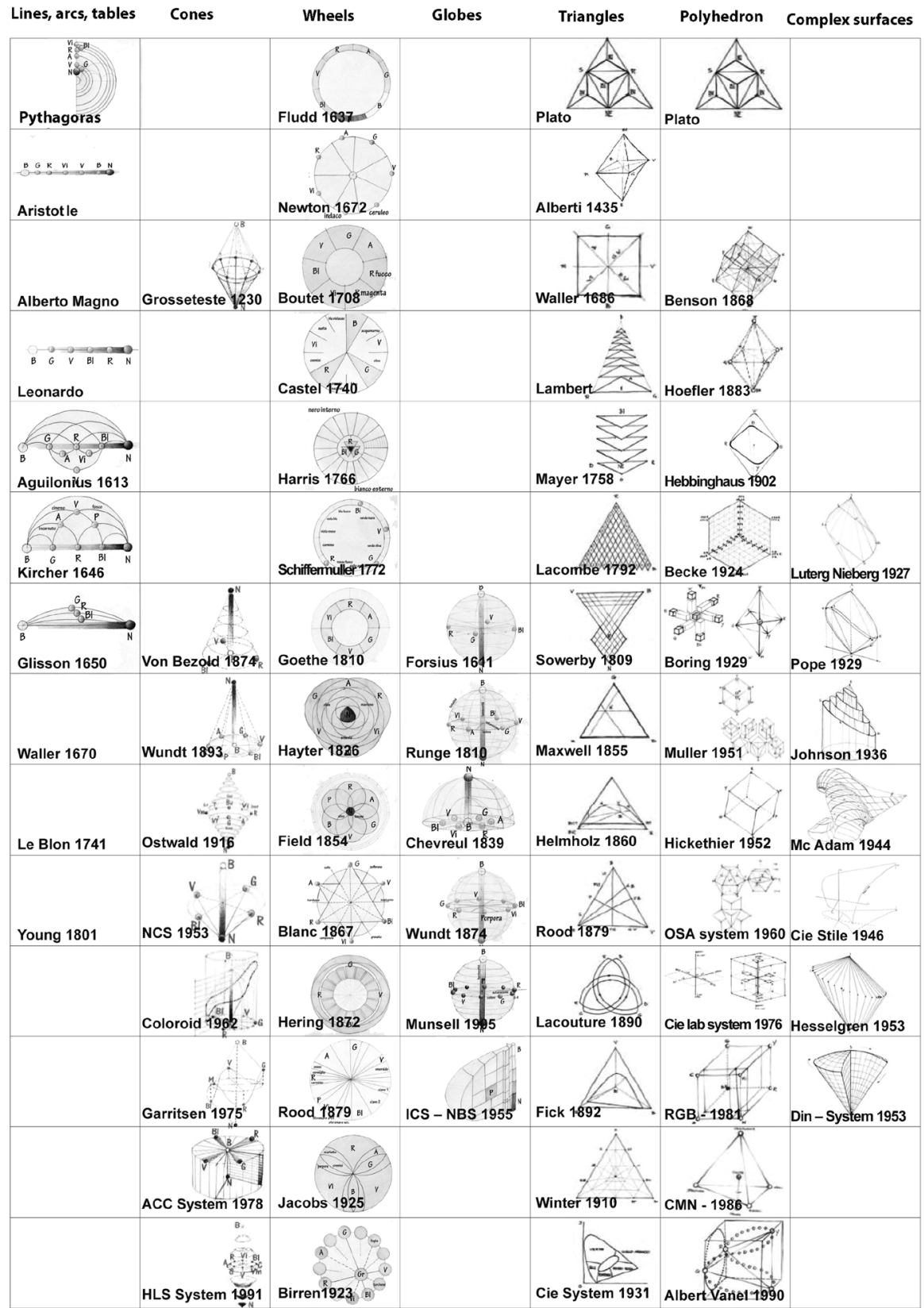

Fig. 1 Chronology of the main color models. The first color models were theoretical explanations without any practical application; from the time of the Renaissance the arts applied an increasing attention to color. In the following centuries the increasing attention to this phenomenon involved natural sciences: physics, medicine and chemistry, with several applications to industry. These applications concerned color printing and dying in the textile industry 
was the starting point of the research and the "joining-ring" to previous works. It was not the goal of the research, but the fixed starting point to understand the role of geometry in color practice (Rossi 2012b).

The research produced a layout of a color solid with changeable geometry, whose surfaces could adapt to several geodesic tessellations (Buratti 2012a). Each regular polyhedron allowed the integration of different regular and half regular shapes by projection onto the sphere. Therefore the quick choice of different sets of colors with analogous harmony and digital representation tools allowed any topological deformations. The model may open its basic features in the shape, the primaries number or in the harmony. This virtual model joins together rooted concepts with present-day needs, stressing the actuality of history and mathematics, whose numeric or geometric models still explain nature's laws.

Since earlier times, the wonder of colors expresses an important role in the approach to exterior experience of the world. Following the nature's examples, the color represents the first featured evidence. Therefore, its main reference is the aesthetics of things outside appearances, not to the visible expression of their substance. In fact, the western culture relates substance to the shape and conceives the measurement - the geometry - as one among the most effective instruments in the knowledge of the physical world (Silvestrini and Fischer 1997; Marotta 1999).

Nevertheless the marvel of the variety and the beautiful harmonies of color led to several efforts to explain the phenomenon and its theoretical concepts, mainly through geometrical references. Following Plato's intuition (a tetrahedron balancing colors and light) the scholars of color applied relations within regular shapes to describe chromatic harmony.

Through the centuries, plane and 3D figures (polygons and polyhedrons) were a concrete reference for theoretical concepts, offering as well a measuring tool for the screening of pigments mixtures, which had several application in painting and in applied arts, such as, first of all, dying and color printing. For example, the introduction of spatial references allowed the quick development of practical applications to painting and dyeing, then to printing. The reference to geometry is a permanent characteristic of color models, and it may help in the upgrade of representational and design instruments. Even if most people are unaware of the importance of geometry in the theory of color, it was the first scientific tool to approach the topic without any final solution. The evidence is the high number of color models (Fig. 1).

Western culture thinks of color as an attribute of appearance, in contrast to shape which it sees as an attribute of substance. Nevertheless, the association between geometry and color is a recurrent topic, and shape gives substance to color. This is the consequence of the key role that Mathematics has played in the explication of the Universe through simplified models using shape, size and number, meaning proportions. $^{2}$ The association between shape and number that characterizes symmetry and proportion in regular figures expressed the harmony of creation in the "music of stars"; color harmony also referred to Geometrical concepts for rules for the composition of hues. Everybody knows that the creation of hues uses

\footnotetext{
$\overline{2}$ See G. C. Argan's introduction to Goethe's Farbenlehre (von Goethe 1993).
} 
different primaries and follows different laws in physics (light) and in chemistry (pigments), but both of them find explication in analogous shapes. In explaining the color theories, geometry stresses its effectiveness in the visualization of abstract concepts and ensures technical control in the applications of simplified models to practice.

The first step in the research ranked the geometric patterns into formal families, identifying the continuity and "evolution" of the color models and the similarities among different shapes. The history of color theories ${ }^{3}$ does not follow a linear evolution, but it shows the importance of the whole of Mathematics in the theory of color. The main heritage is just the reference to geometry, which dates back to the birth of Western thought in the Greek culture in the colonies of southern Italy.

The history of color theories is well known. Anyway it is worth summarizing the evolution of the color models, in the relationship between the shape elements and color features within the different concepts and their direct fallout in practical applications to art and industry. As everybody knows, the philosophers of the Classical age laid the foundations of the sciences on the study of natural phenomena, searching for a rational explication in the net rules of geometry.

The oldest color theory belongs to the Pythagorean School, who explained vision by means of the rays of invisible "fire" that hit the body's surface. It is what leaves the eye through the air or the water, reaching the objects then returning to the observer, what Pythagoras called $\chi \rho \omega \mu \alpha$ (chroma, the color). Later, Empedocles referred to color as part of experience.

According to Democritus' atomistic school, color is a secondary quality of things, because atoms have no color. Thus ordinary objects, which are made of atoms, have no color. They have a surface structure characterized by size, shape, orientation and order of atoms that allows us to perceive the color as a consequence of the shape. Plato's theory explained the hues as a balance among pure colors on the tetrahedron apex, where he put white black and red, besides what he called "shining"; yellow, blue, green and purple generated on the faces. Aristotle theorized five primary colors, in addition to white and black, derived from the theories of Empedocles, and he ordered them on a line connecting white to black (Fig. 2).

None of these theories was conclusive, and while the Greeks did not manage to formulate a systematic doctrine for the explanation of the phenomena of light, vision and color, yet they provided the basic components to build more complete and consistent systems.

The fall of the Roman Empire pushed the scientific center forward to Western Asia, where Greek colonies had prospered, and it became the vector of the sprawl of the classical heritage in the Arab world. Between the tenth and twelfth centuries knowledge flourished in Bagdad, while Christianity denied the natural sciences as a study less worthy than theological issues. Among Arab writers it is worth quoting

\footnotetext{
3 The scientific study of the color model from the past was the topic of some important works such as those by Silvestrini and Tornaghi (1982), Silvestrini and Fischer (1997), Marotta (1999), Kueni (2003, 2005), Lowengard (2006) and others, who approached the topic with a mainly historical interest. The great persistence of the geometical reference justifies the comparison of the shape recurrencies of the models and their specificity. On one hand, different shapes express similar concept, and on the other hand, similar shapes can recall opposite assumptions, just because of the great adaptability of the concept to the practice.
} 

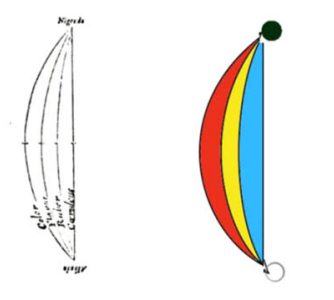

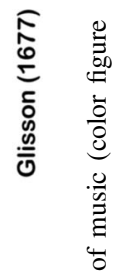
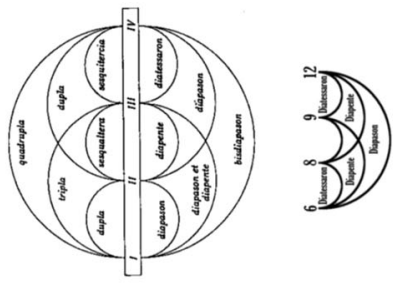

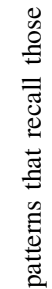
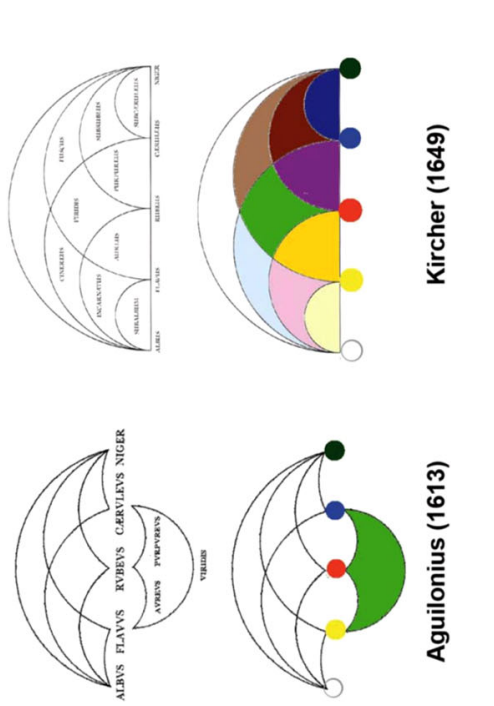

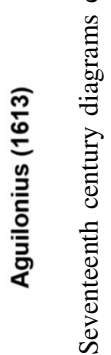
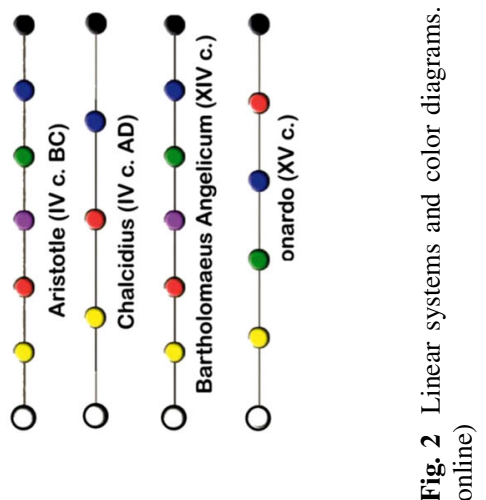
Alhacen (Ibn al-Haytham), who around the millennium developed a color theory with a mathematical, physical and physiological approach. He overcame the limitations of Aristotle, Galen and Euclid's theories with the experimental demonstration of important optical principles.

Around 1,200, the corpus of optics literature was translated from Greek to Latin. The studies from the Classical age caused a great deal of attention to be paid to natural phenomena, firstly among the Franciscan monasteries of Canterbury, London and Oxford. A vivid scientific culture grew up, thanks to the spread of the works of Al-Kindi, Avicenna, Averroes and Alhacen. Robert Grosseteste, who was the first rector of the Oxford school, and Roger Bacon studied the optical treatises of Euclid and Aristotle. Grosseteste approached the vision of color through optics and perspective; following Plato's teaching, he stated that the explication of color requires a three-dimensional model and he proposed a double cone with pure colors on the equator and black and white on the apexes, confirming color as a balance between light and darkness (albedo and nigredo) (Silvestrini and Fischer 1997; Marotta 1999).

\section{Geometry Between Theory and Practice}

Medieval optics was similar to the classical approach that dominated until the sixteenth century, when the Renaissance redeveloped the previous knowledge in the arts. Leonardo da Vinci (1452-1519) in his 'Treatise on Painting' speaks about "simple color" to create all other colors, in addition to black and white: these were red, yellow and blue. To better visualize the concept, Leon Battista Alberti suggested the shape of a double square pyramid, with pure colors on the square's apexes. Besides perspective, colors were also a concern of painting and both of them applied geometry to theory and practice!

In 1666 Isaac Newton reinterpreted Aristotle's optics in experimental physics. He studied the relationship between light stimulus and color perception and formulated a new color theory, which was based on the mixture of light. He stated that color could be represented with a mathematical model. In facts his color wheel was applying old symbolic patterns, such as the choice of the number of colors. Certainly Newton, who grounded his theory on a scientific basis, identified seven main colors in his chromatic scale and closed the extremes in a wheel as Fludd had done in the previous century, despite the obvious differences of the wave's length on the two opposites in the spectrum of visible light.

In the eighteenth century Mayer successfully organized colors in relation to the mixing of pigments, creating the key element of all the subsequent color theories, a regular triangle with three primary colors (red, yellow and blue) on its vertexes. He applied algebra to measure the mix of pigments by three factors. He supposed the couples of complementary colors as the basis of any color harmony.

His heritage is grounded in Goethe's theory, which was re-affirmed by Itten in the twentieth century (Rossi 2012a). All of them stated that color's harmonies are based on the geometrical measurement and balance of nuances, and that they are 


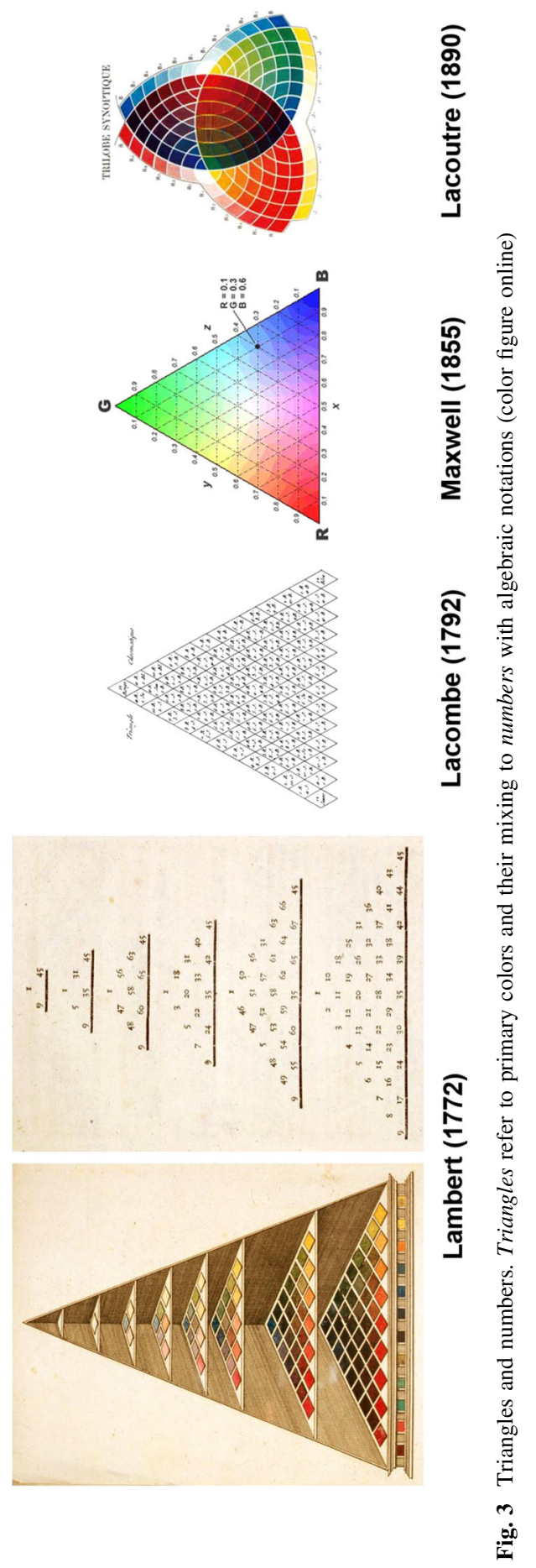


M. Rossi, G. Buratti

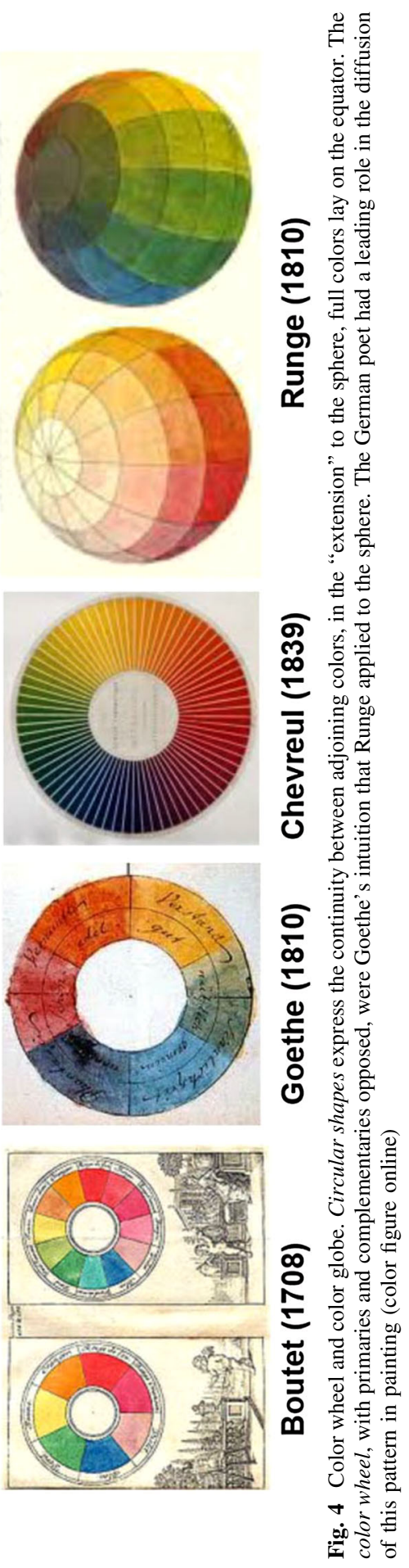



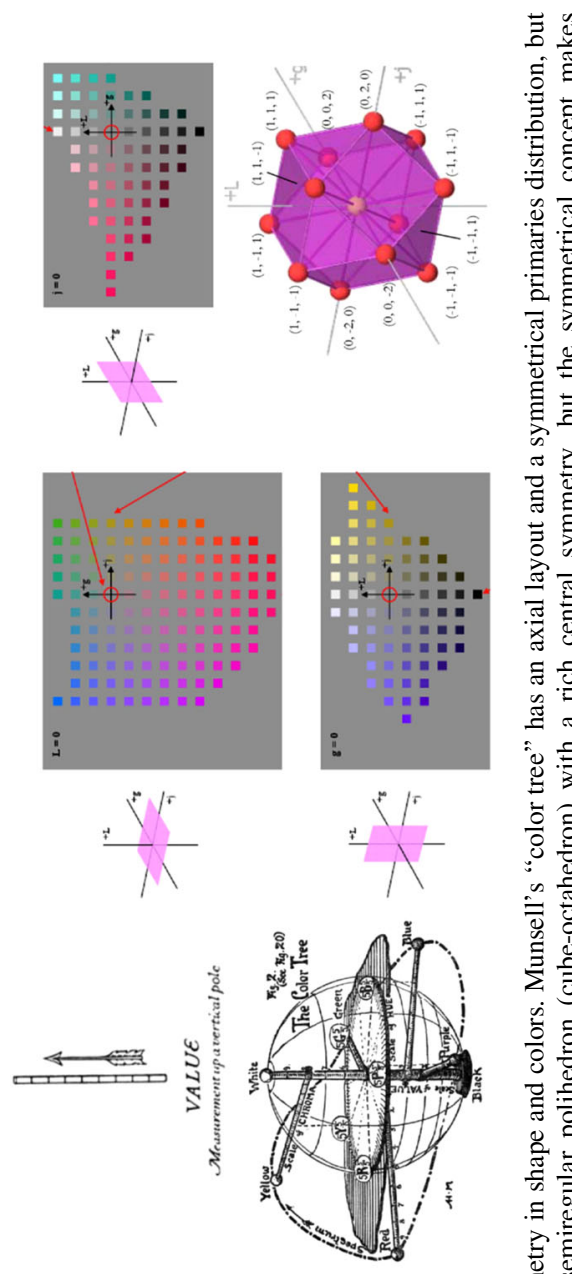

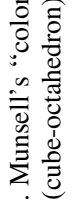

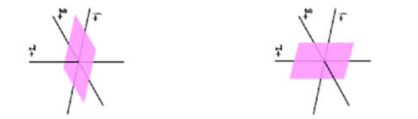
흥 홍

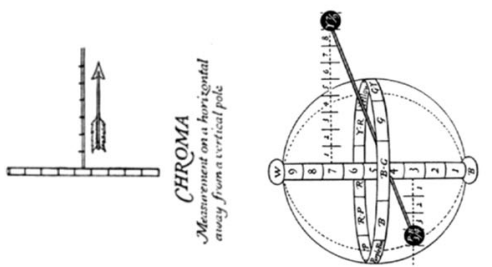

है

的。

富育言

政

离完 言章.

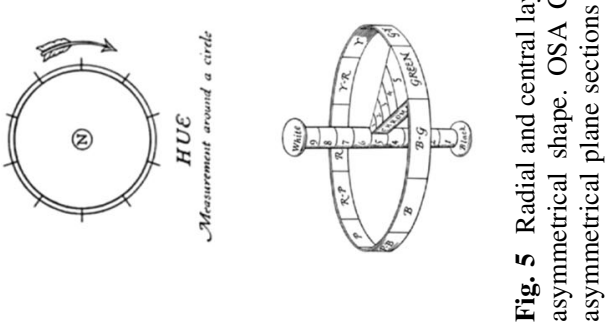


still the most relevant attempt to find an aesthetic rule for color composition in Art and Design (Fig. 3).

Newton's color theory, based on light waves, did not concern painting. According to Goethe, he made "one of the most harmful mistakes". On the contrary, Goethe's theory concerned only the colors obtained with pigments. The German poet developed the concepts of physiological, physical and chemical colors with empirical studies that are the basis of his color theory. The debate with Schiller and the correspondence with Runge contributed to the formulation of geometrical models that had a stark influence on peinture theory and practice. Goethe adopted the color wheel to explain and measure pigments. Runge developed it into a sphere, but none of them were really original (Runge 1810). Since thousands of years ago, painters have known that they can get all the colors starting from just three pigments: red, yellow and blue, although in practice they sometimes needed much more to better characterize the hue (Fig. 4) (Rossi 2012b).

In the nineteenth century Hermann Grassmann introduced a mathematical model of geometric color that led to the colorimetric theory and the full review of the layout of the spectral colors of Newton's wheel by James Maxwell (1831-1879).

In 1855 Maxwell perfected the location of spectrum color around the circle, and therefore the theory of compound colors, through actual experiments on the mixture of colors. Maxwell started from the consideration that the work of Newton-the arrangement of colors on the circumference of a circle-was only a temporary arrangement, and that the correct relationship of the colors in the spectrum required direct observation (Briggs 2013).

Colorimetric applications have common use in the compilation of atlases and color systems, such as those of the American painter Albert Henry Munsell (1858-1918), that develop their own three-dimensional color order based on the perceptual attributes of clarity, hue and chrome. Similar systems are NCS (Natural Color System), DIN and OSA System. The regularity of the concept adapts to different extensions of color hues, making irregular shapes with axial or polar references (Nassau 1998) (Fig. 5).

The Nobel Prize winner Erwin Schrödinger understood that the tristimulus can be associated with a Euclidean metric and that the same perceived color differences do not match equal distances. In other words the tristimulus space does not have uniform scales. He proposed an equation that provides the color space of the Riehmann geometry, such as in Einstein's theory of relativity. This space has four dimensions, while color has three coordinates.

Indeed color discrimination is related to the second stage of vision and therefore it should be represented in a different space from tristimulus-which represents the first stage-derivable from non-linear transformations. In practice this would happen in the CIELAB (1976) space, but it is widely moot if this space does really describe the process of color vision (Spillman et al. 2010). 


\section{Color's Concepts and Patterns}

Color theory shows ties with geographical areas, with specific disciplinary purposes and with different techniques. It highlights the application of the different systems as well as the inability to find a definitive model able to satisfy both the physical component (lights) and the chemical one (pigments) of the phenomenon. The main theories identify a small number of primary colors. Geometry's rules fix their position in specific points of the model, which identifies a shape. The mixing of pure colors creates all the visible nuances, and mathematical models have provided the measuring tool in pigment mixing in dyeing and painting from remote times up to the digital screen.

Models never reached an absolute solution and no shape in the formal sample is contingent. This evidence stresses the difficulty of a single representation of the
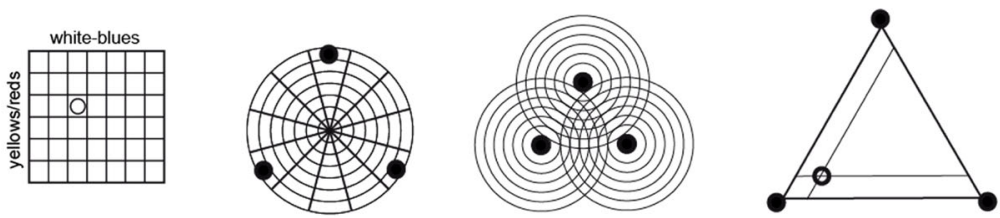

2D patterns (cohordinates and color mixing)
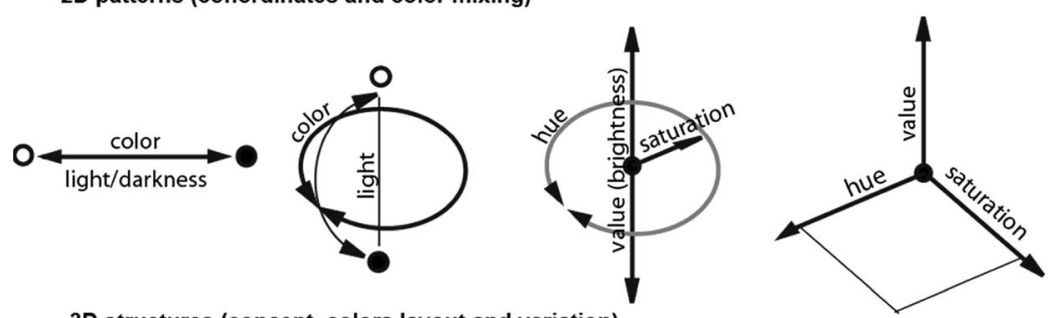

3D structures (concept, colors layout and variation)
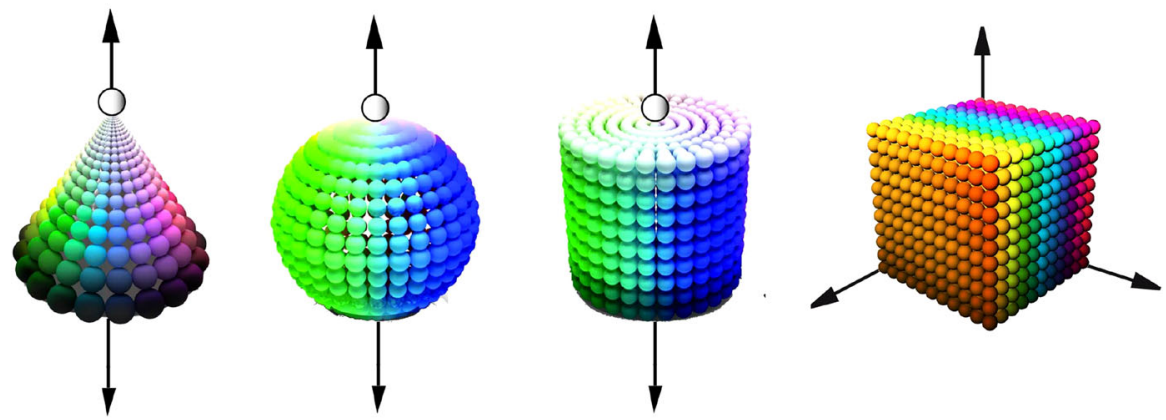

Fig. 6 Patterns and structures. The closing of linear organization of colors joining black and white led to color wheels and to rotation shapes with the extraction of white and black (albedo and nigredo). The main 3D structures apply the same rotation or translation layout, no matter the primary number. Rotation volumes have a similar concept but their shapes stress colors with different emphasis, enlarging some of them. The double-cone stresses full colors with the discontinuity of its surface; the sphere exalts continuity in color variation; in the cylinder, the surface discontinuity stresses the difference between real colors and achromatic colors. The polar axis that links black and white is the common feature allowing the topological transformation between different shapes (color figure online) 
complexity of color. Number and quality of the primaries are the hallmarks of any color theory; the shape is the first discriminator, because it explains the hierarchy among the different elements. Cataloguing, with respect to the number and the nature of the primary colors, shows that analogous concepts coexist based on entirely different assumptions (Fig. 6).

The models can be grouped into families and "clans", according to their shape:

- circle, triangle and square;

- $\quad$ sphere and cylinder; cones, double cones and pyramids;

- cube and other regular polyhedrons.

The comparison shows the recurrence of similar patterns on different shapes, and of different concepts using the same shape. Beside raw figures, in the second half of the twentieth century complex surfaces, algebraic structures and other mathematical models also appeared. This last group contains mathematical models with rather complex shapes that integrate objective parameters related to perception, influenced by physiological factors. In the last decades of the twentieth century some multidimensional models appeared, such as matrixes, which allowed the implementation of artificial intelligence systems, but later simple shapes reappeared in relation to digital applications. Different shapes show an evolution that was not linear, and a different attitude in practice.

The comparison among models depending on the form, on the cultural environment, on their theoretical field or on their practical applications emphasizes the stark persistence over time of similar concepts and symbolic references, as a proof of validity. Model families do not exhibit any evolutionary development,
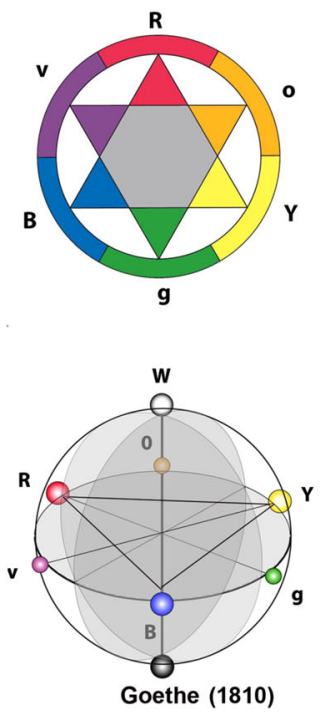
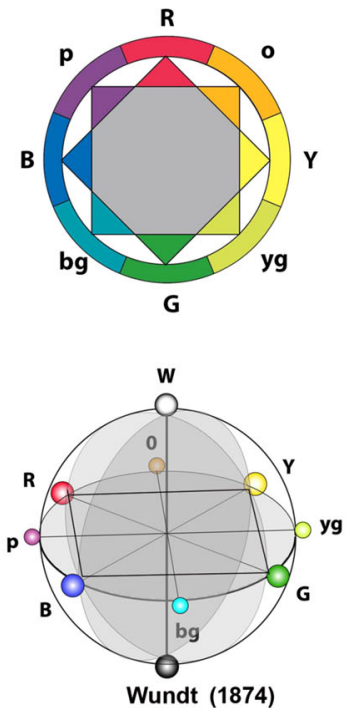
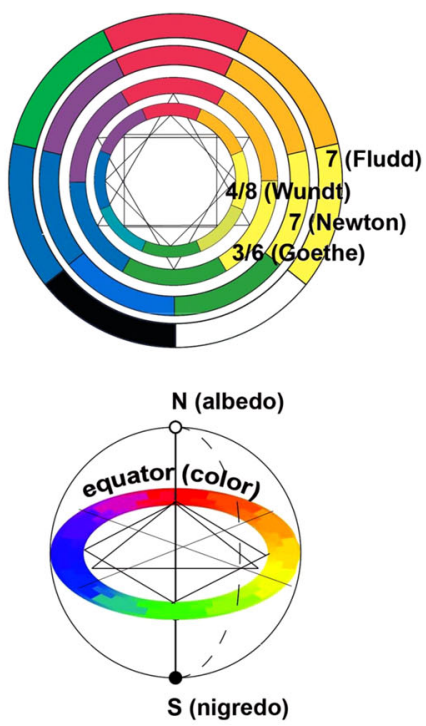

Fig. 7 The sphere. The first color model that referred to the sphere was Forsius's (1611), similar in color layout to Wundt's one. The number of primaries (or principal colors) rules the extension of cold and warm colors and the opposite hues (color figure online) 
although it is possible to recognize a refinement process within the same group. The comparison reveals some recurrent features and the persistence of some symbolic, astrological and alchemical references, which stress the traditional derivation of chemistry, such the vertical axis of the achromatic line (albedo/nigredo) which is almost always provided with light top and dark bottom. The number of primary colors in different systems (3, 4 or 7) shows an unexpected relationship with symbolic elements that recurred in Western and Middle Western traditions (Fig. 7).

The comparison shows the alternative possibilities of articulating the model with continuous or discontinuous surfaces, emphasizing the gradualness of color, rather than the need to discretize the reference pitch. With the language of digital modeling, these models respectively refer to NURBS or mesh.

The simplest forms still have practical applications: the RGB color model is just a cube! Recent models emphasize the validity of many theories of the past, supporting the aim to apply geometry to color in the development of digital design tools.

\section{A Geometric Tool for Color Design}

The research has developed a digital color model that would allow the immediate visualization of equivalent color combinations - i.e. with the same symmetry with respect to the chromatic balance, according to Goethe and Itten, through the rotation in the space of a number of radial elements (pointers). This requires the insertion of a regular solid into the sphere, and surface tessellation to apply the symmetries of the color. The geodesic tessellation allows the smallest deformation of the modules. The descriptive geometry offers then a theoretical solution by central projections; the graphic potential of generative software facilitates the modeling and the tessellation of any surface: the geometry gives the concept, while digital tools develop it.

A famous reference is Buckminster Fuller's architecture, in which the shape "is" the design solution. He applied sphere geometry and symmetries of regular solids when looking for innovative solutions to construction problems, with a very personal rethinking of nature's models. He implemented the symmetries of the regular polyhedrons that Plato put at the base of reality. The properties of the geodesic sections, which Fuller developed in his Dymaxion's map projection for air navigation, before the patenting of light domes, can be experienced in the representation of color.

Among regular solids, the octahedron seems suitable to digital color, because it joints the primary of the RGB screens with the pure color of pictures and the two achromatic colors. This regular shape responds to the actual conditions; its six vertices bear the four psychological primaries (red, green, blue and yellow) and the two achromatic colors (black and white) in a prominent position, entering all the gradations in between them. The choice of geodesic lattice and the reiteration of the same generative algorithm play down the deformations of the tiles. Colors lie only on the surface to show all of them, the saturation can be controlled through the transparency filters that are in the main graphic software. The color layout is similar 
to the double cone of the NCS system, which recalls the fundamental models of the twentieth century, such as Hoefler's, Hebbinghaus's and Boring's, and also Wundt's sphere, that have a similar organization of the pure colors.

Such as any regular polyhedron, its surfaces are suitable for the geodetic projection on the sphere. The generative modeling operates by linear criteria: there are input data, processing, and output. In the construction of the color-model, the starting point is the implementation of an algorithm that defines the equilateral triangle corresponding to the face of the octahedron (Fig. 8).

The manual distribution of colors into the tessellation applied the arithmetic calculation of the tiles' color values through the RGB coordinates in their gravity center, and led to the digitisation of hues on the tiled surface with Adobe Photoshop. To maximize simplicity, the edge of the test-model connecting to two adjacent primaries (and/or the spherical segment), was divided into five parts. The digital coloring verifies the correctness of the geometric concept (Figs. 9, 10).

Finally, generative software developed the digital modeling of the polyhedron and the tessellation of its surface. The geodesic projection on the sphere adopted the pattern that ensured the higher regularity in the dimensions and shape of the color tiles. The work was carried out with Grasshopper, a Rhinoceros plug-in that translates the programming syntax in a friendly interface, expressing the ties between the algorithms in a diagram. The parametric software allows the management of complex objects, by a series of repeated steps that permit the decomposition of the problem into a finite number of simple tasks. The basic design elements are interconnected algorithms; at each cycle they influence each other, generating step by step other elements with a higher level of complexity, until the final geometry.

The software implements the modeling of a parametric color-solid, by further processing of Euclidean shapes by "automatic" transformations between topologically equivalent tiles. Grasshopper manages the parametric control of the color; it adds the possibility of applying different operators to the modeling, imposing successive transformations to the starting element through repeated algorithms. Besides the control of the shape deformations, it allows the automatic discretization of chromatic scales, by applying the parametric process to color rather than to form. Eventually it creates a digital representation of a complete color-system with a few

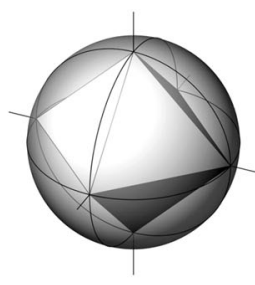

(a)
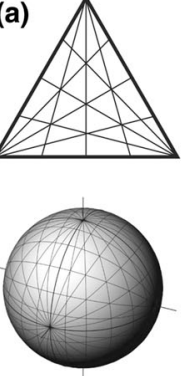

(b)
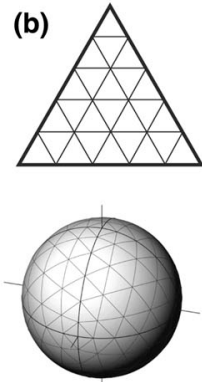

(c)
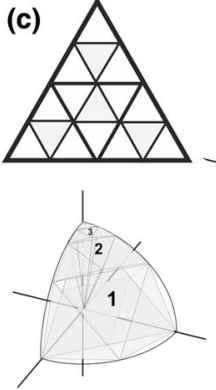

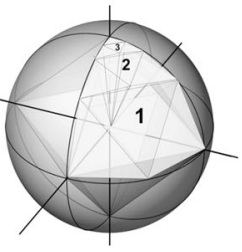

Fig. 8 Octahedral tessellation on the sphere: a geodesic, b triangular, c reiterate tessellation. The tessellation with regular shapes minimizes the deformation of spherical tiles 


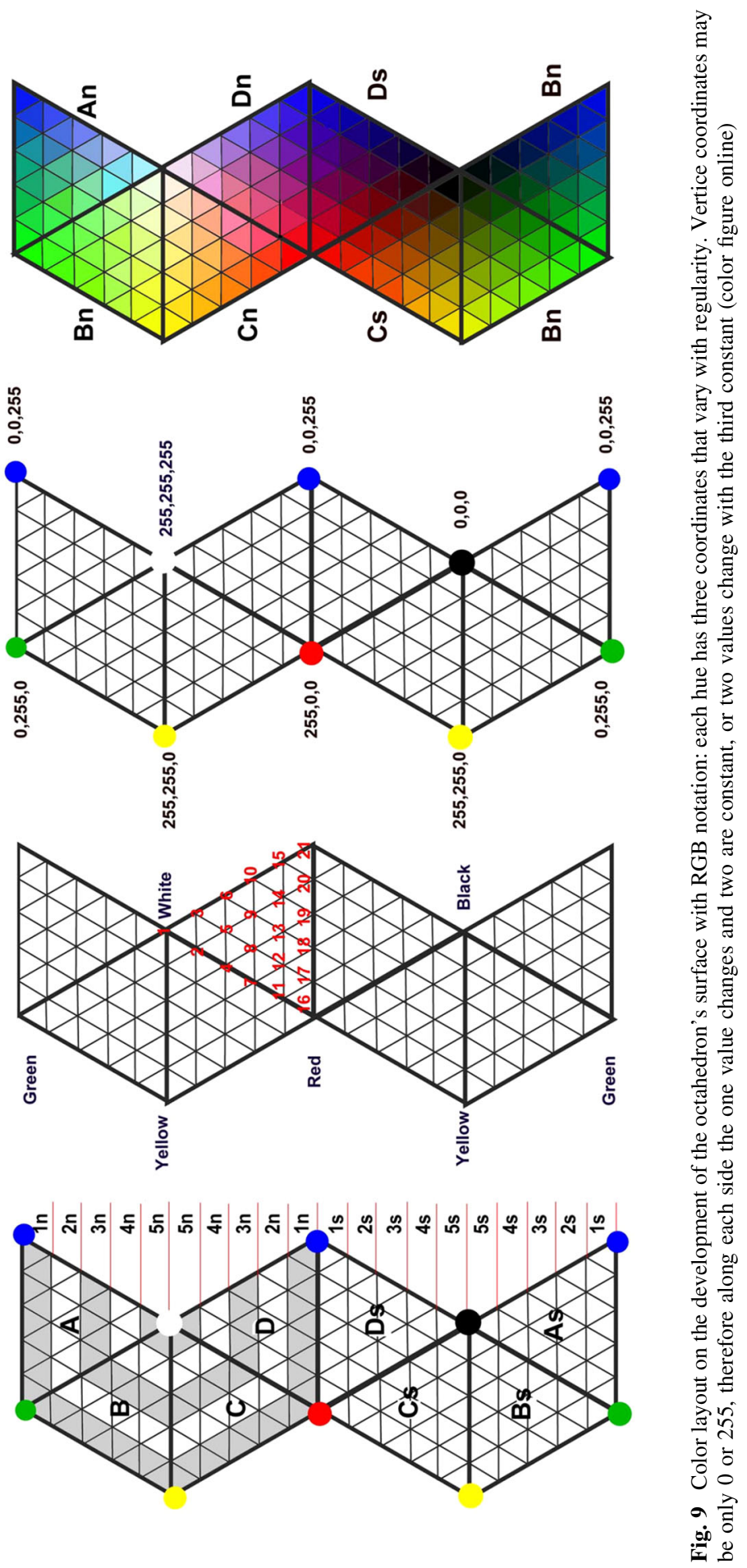


side $\mathrm{D}$

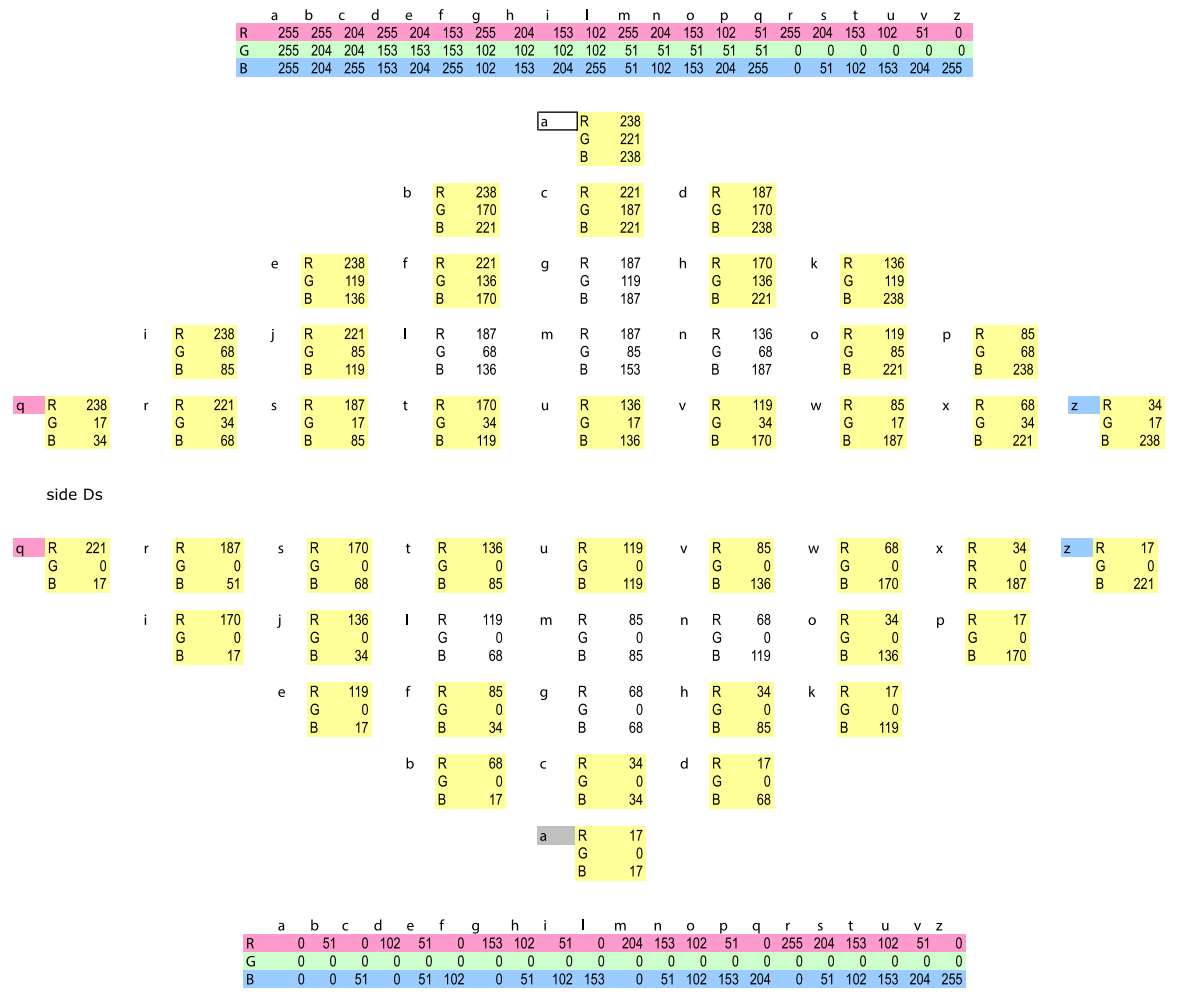

Fig. 10 The manual coloring calculated the RGB coordinates of each tile vertex, then the tile's color as the average of three. In both cases the arithmetic measurement of color shows regular variations of the three "numbers" that can increase, decrease or stay without any full mirror symmetry (color figure online)

fundamental operations. The control of two independent variables is easy and intuitive, while the parametric control of a third one requires more complex algorithms, not immediately available.

The first modeling regarded shapes, not the color mixing of the pure colors (Fig. 6). The color modeling changed with the shape: the generation and the coloring of sphere, cone, cube and cylinder do not have the same complexity. In any case, generative software manages the transition from one solid to another, changing the shape with topological deformation. As well, the position of the primary colors on the model can be controlled with a few elementary operations, through the replacement of the original geometry or by changing the gradient of color.

The following step is the addition of the algorithm that divides the faces into the number of triangular tiles, required to show all the different shades of color in the model (Buratti 2012b). Next is the tessellation of the sphere by a stereographic projection; in such a way all the sides of the triangles lie on maximum circles. The relationship and the basic parametric functions are fixed, while the number of tiles and colors can be changed as required (Figs. 11, 12). 

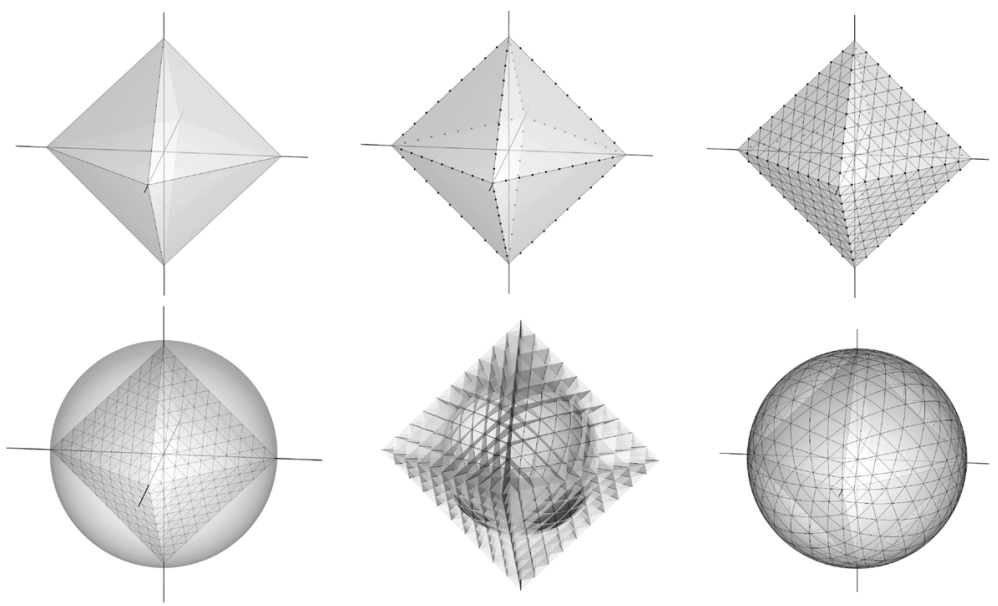

Fig. 11 Generative tessellation on the spherical surface with Grasshopper. Since the dihedral angle of a regular octahedron is arc $\cos (-1 / 3)$, it is possible to obtain it by rotating and tilting the solid. The algorithm transfers all the tiles on the octahedron faces on the sphere by central projection with their color codes. The digital solution of the regular variation is possible with Grasshopper's tools. The division of the edge into 12 parts would provide a total of 1,152 three-components hues on the faces, which with the 6 pure colors and $12 \times 8$ two-color shades would forms a palette of 1,258. Larger quantities of hues require a high increase in the RAM memory to avoid operations working too long on the machine and the instability of the system. In fact the projection of spherical triangles by planes intersecting the sphere and the attribution of color to the tiling need a high number of calculations and this increases geometrical proportion in relation to the edges' modules
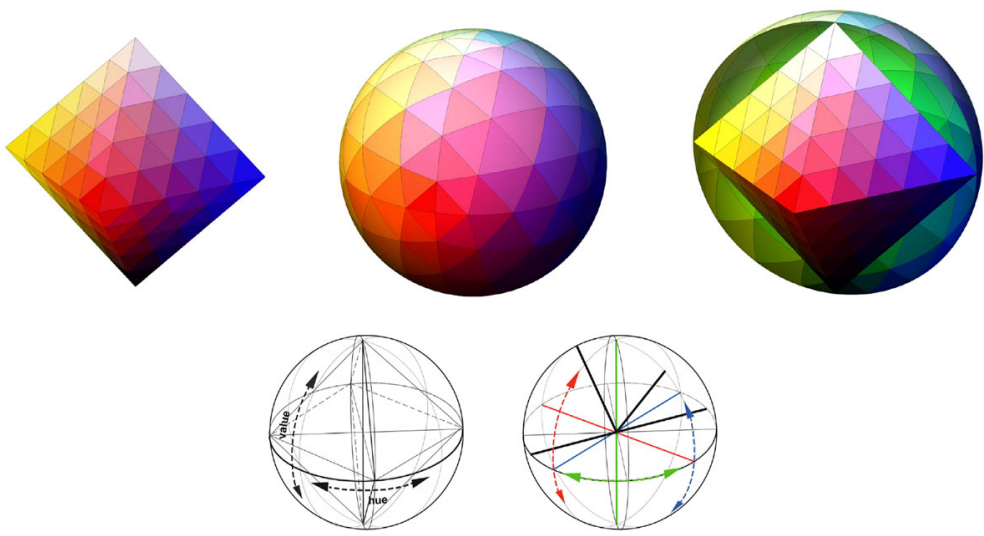

Fig. 12 The digital model and the color-design tool. Octahedral tessellation. The color code is based on the distance between each block and its attractor points, corresponding to vertices of the solid. Despite the theoretical possibility of obtaining an unlimited number of hues and tessellations, the first test restricted the number to 25 modules in each face. The following model has therefore 200 three-components hues, depending on the RGB system that is in the tessellation on the faces, besides 40 shades of two colors along the edges and 6 pure colors on the vertexes that have not any visibility in the tessellation because they correspond with immaterial elements (the line and the point). A set of pointers inside the sphere can select variations of hues with similar harmony. The algorithmic generation of hues is easy because of the linear changing of coordinates (color figure online) 
The representation of color follows a logical relationship with geometry and several Grasshopper components can manage it. Then any regular shape can rotate inside the sphere, such as any set of pointers-no matter how many and their orientation-and they easily select color series with equivalent harmonies. The model refers the color hierarchy to the number of components in each hue-tile. Pure colors lie on the vertices, while two-color shades are along the edges: the tessellation does not show them because they don't have any material extension as geometrical entities. Further transformations are required to make tiles for pure and double colors, smoothing out edges and vertices.

The coloring is bound to the color spectrum of the software. Therefore it is conditioned by color generation on the screen and the digital modeling applies the RGB system, which is that of the display. If the colors of the other tiles vary according to their distance from these points and they are defined by three integers between 0 and 255, representing the colors Red, Green and Blue in the RGB system, then the process should keep some symmetry of the three primary colors in RGB screens.

Color coordinates show regular increasing/decreasing in their RGB components, but without any hard symmetry as they have in the RGB cube. The cube shows in its vertices all combinations of color coordinates, while in the octahedron the cyan and the magenta "disappear" (Fig. 13). Therefore the hues number gets smaller and opposite colors do not show any symmetry in their coordinates. The cube is the dual solid of the octahedron, but there is duality in the color-models, because there is "numerical symmetry" in the color notation: the octahedron can't show all RGB colors, with a limitation in the cyan-magenta hues. A continuous transformation is possible only with different movement in primaries (Fig. 13).
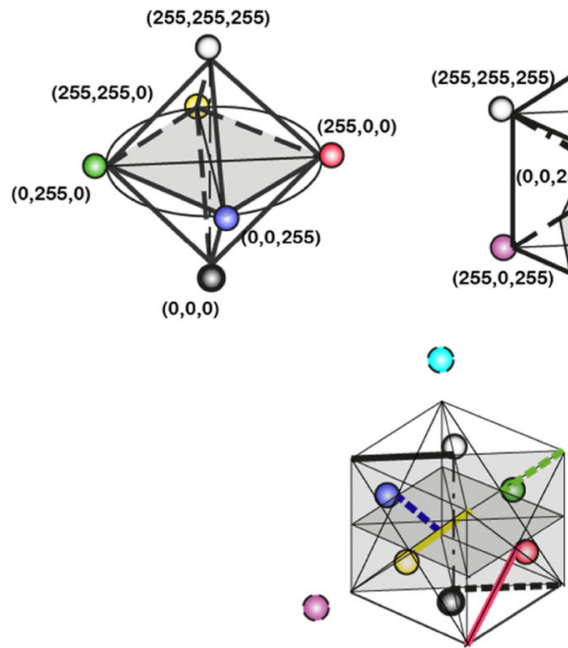

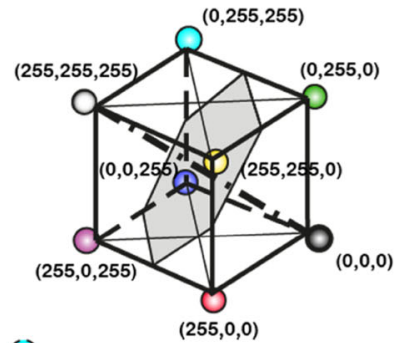

$(255,0,0)$
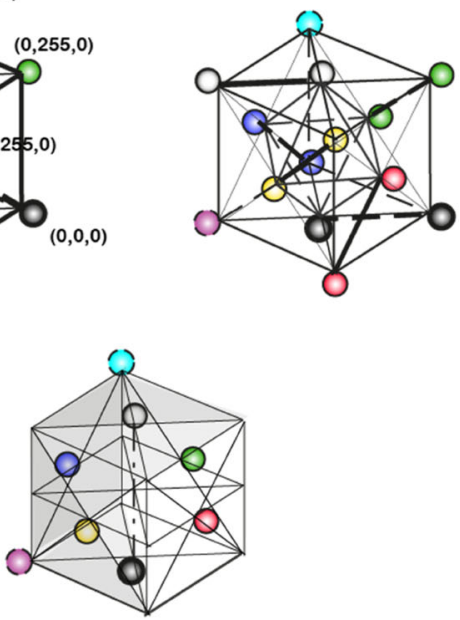

Fig. 13 Color and shape. Colors layout in the octahedron is different to that in the cube and it is impossible to generate the one from the other. The "movement" of opposite couples of pure colors follows different rules of symmetry. Cyan and magenta "disappear", therefore it is impossible to show all RGB hues on the octahedron, with a strong limitation of the cyan-magenta area (gray on the bottom-right cube) (color figure online) 

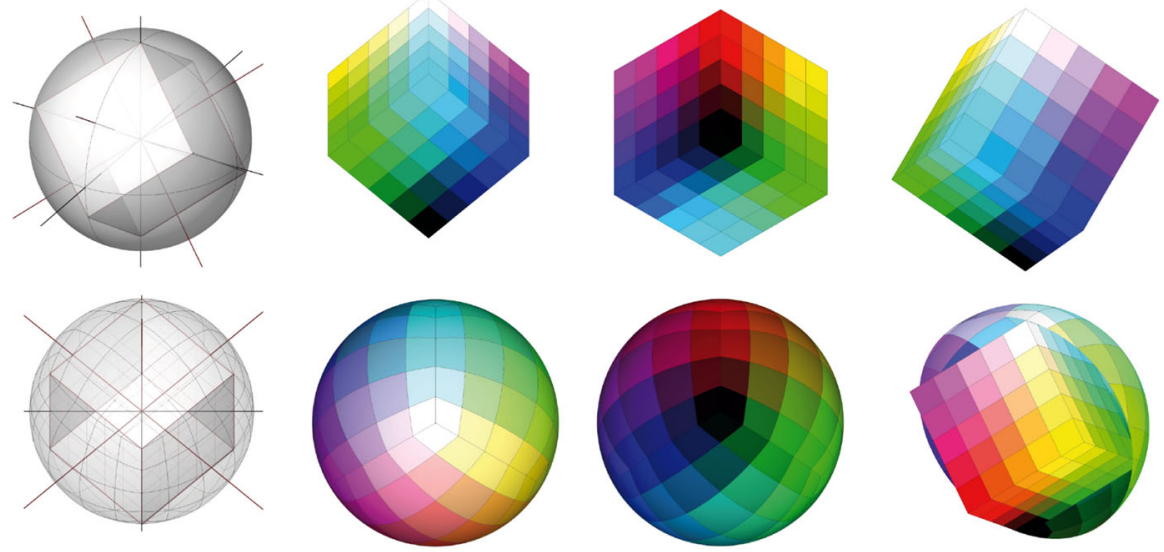

Fig. 14 Cubic tessellation on the sphere. Along sides change only one among the three RGB coordinates; the central projection generates a tessellation with larger differences among tiles in shape and area; the calculation of hues is longer because each color value is the average of four. The digital generation requires longer calculation because of the lack of suitable Grasshopper operators (color figure online)
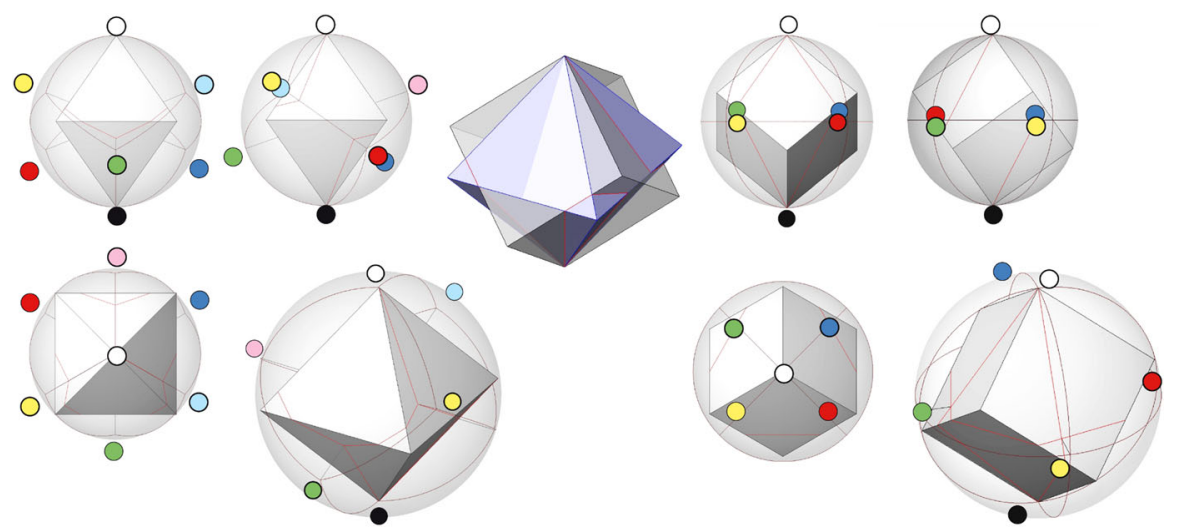

Fig. 15 Duality in shapes, not in colors. With black and white as a common vertex two opposite faces share the same plane and complementary colors lie on a mid-line of the octahedron's faces; other fundamental colors have "generic" positions on the dual shape. The projection of each shape on the dual one produces a regular layout of colors, but there is immediate correspondence with the featuring elements (color figure online)

Different managements of colors on the sphere are possible with a different starting layout and therefore a different tessellation. A palette with all RGB colors is obviously suitable starting from the cube, but it is "incompatible" with the octahedral lattice, because the two shapes don't have the same symmetry and they cut without any full symmetry (Fig. 14).

Further projection of each tessellation on the dual shape (the cube on the octahedron or the octahedron on the cube) is possible: colors layout is "quite" regular but it leaves any immediate correspondence with the shape elements. Actually the octahedral genesis produces a reduced palette but its tessellation by 
equilateral triangle has less deformation on spherical surfaces, the cubic one has the full RGB palette, but higher deformation in its further tessellation (Fig. 15). This means that the two shapes make different color harmonies, and the digital selection tools would generate different color sets. The octahedron appears to be more suitable to the color-design tool.

\section{Conclusion}

A common reference to geometry stresses the keen relationship between color and architecture, both of them applying measure and proportion as a rule of harmony. History emphasizes the use of geometric models to rationalize the phenomenon of color. The references to geometry date back to the ancient world, as a consequence of the classical principle that conceives reality as a harmony of shapes and numbers.

Models show the persistence of some basic patterns. In the wheels the symmetry emphasizes the relationship between the primary colors and the pattern. The relationship of color to geometric entities displays different concepts through similar shapes, because the most important factor is not the shape but the very essence of geometry.

Two broad groups gather 3D shapes: continuous or discontinuous surfaces favor respectively the gradual (and theoretical) transformation of color rather than the discretization of nuances for practical purposes. The elements of the geometric structure (axis, apexes and edges) show their own features in each color-model. The quality and the position of primaries affect the spectrum of both warm and cool colors. In continuous shapes, such as the circle and the sphere, it can be corrected, as Birren did in the asymmetrical arrangement of his 13 main colors. The sphere plays the "neutral" shape in the topological transition between these two groups and among different shapes. The last ones offer different width to the colors range: the cone and the sphere shrink the gradient at the poles, pointing out that the approach to the achromatic reduces the nuance gradient. The variation is linear in the cone, while in the sphere it increases gradually and the phenomenon gets more evident. Digital representation increases the importance of color in design and it provides new tools for color design that find their roots in the history of color models.

On the other hand, the variety of color models tells of the development of Mathematics, starting from the musical harmony in arithmetical relations of linear systems or the proportion of regular shapes, up to algebraic geometry in topological surfaces, Graphs and digital algorithms: geometry is changing along with the times, but it still offers a suitable reference for color applications.

Eventually color combination is not a science but an art that-such as geometry-builds a logical architecture on its own postulates. Color postulate is the choice of the number of primaries and their quality. Changing the geometry changes the "harmony" of colors. 


\section{References}

Briggs, D. 2013. The Dimensions of Color. http://www.huevaluechroma.com.

Buratti, G. 2012a. Generative Algorithms and Associative Modeling to Design Articulate Surfaces. In: Relationships Between Architecture and Mathematics. Proceedings of the Nexus Ph.D. Day, ed. M. Rossi. Milano: McGraw-Hill.

Buratti, G. 2012b. Digital Color Harmonies: A Prototype Color Harmonization for Smartphone. In: Descriptive Geometry and Digital Representation: Memory and Innovation, ed. M. Rossi, 99-105. Milano and New York: McGraw-Hill.

Kuehni, R. G. 2003. Color Space and its Division: Color Order from Antiquity to the Present. New York: John Wiley and Sons.

Kuehni, R. G. 2005. Color: An Introduction to Practice and Principles. Hoboken, New Jersey: John Wiley and Sons.

Lowengard, S. 2006. The Creation of Color in Eighteenth-Century Europe. New York: Columbia University Press, Gutenberg-e.

Marotta, A. 1999. Policroma, dalle Teorie Comparate al Progetto del Colore. Torino: Celid.

Nassau, K. 1998. Color for Science, Art, and Technology. Amsterdam: Elsevier Science.

Rossi, M. 2006. Nature's Architectures and Built Forms: Structures and Surfaces Between Idea and Design. Nexus Network Journal, 8 (1).

Rossi, M. 2010. Geometry, Shape and Color in Design: Reseach Notes from Historic Color Theory. In: Color and Light in Architecture, ed. P. Zennaro, 519-525. Venezia: Nemesi.

Rossi, M. 2012a. The Practice and the Grammar. Theory of Color and its Representation in Design. In: Elogio della Teoria. Identità delle Discipline del Disegno e del Rilievo, eds. Laura Cerlevaris and Monica Filippa. Roma: Gangemi Editore.

Rossi, M. 2012b. The Geometry of Color. Shape as a Measuring Tool. In: Descriptive Geometry and Digital Representation. Memory and Innovation. General Investigator R. Migliari, ed. Michela Rossi, 189-200. Milano: McGraw-Hill Education.

Runge, P. O. 1810. Die Farbenkügel. Zurich.

Silvestrini, N. and E.P. Fischer. 1997. Color Systems in Art and Science. Flammarion: Paris.

Silvestrini, N. and A. Tornaghi, eds. 1982. Colore: Codice e Norma. Bologna: Zanichelli.

Spillman, W., M.V. Schindler, S. Wettstein, and I. Haupt. 2010. Farb-Systeme 1611-2007. Basel: Schwabe Verlag.

von Goethe, J. W. 1993. Zur Farbenlehre (1810). Tübingen. Italian translation ed. G.C. Argan, Milano: Il Saggiatore.

Michela Rossi is an architect and Ph.D in Architectural Survey And Representation; she is associate professor in the School of Design-Politecnico di Milano, where she teaches Design Drawing. Previously she has been in the faculty of the schools of Architecture in Florence and Parma.

Giorgio Buratti is a Ph.D student at the DIREP unit (Design and Representation) in Design School at Politecnico di Milano. He graduated with top marks from the same university where he obtained a master's degree in ergonomics too. 